\title{
Below-knee amputation for Charcot joint developing 40 years after spinal cord injury. Case report
}

\author{
O Yoshimura MD, T Toyonaga MD \\ Division of Rehabilitation Medicine, Kyushu Rousai Hospital, Kokuraminami, \\ Kuzuhara-Takamatsu 1-3-1, Kitakyushu City, 800-02 Japan.
}

A Charcot joint developed following a spinal cord injury in a patient who had
sustained a fracture-dislocation of the 12 th thoracic vertebra and a spinal cord
injury in a cave-in accident in a coal mine 40 years previously, and had since
been assisted in walking with the aid of a short leg brace and a cane. Recently,
the patient developed Charcot joints of the right knee and ankle, and the right
ankle joint also became infected with a refractory open wound necessitating a
below-knee amputation.

Keywords: spinal cord injury; paraplegia; Charcot joint; below-knee amputation.

\section{Introduction}

The arthropathy complicating neurological disorders where there is chronic progressive degeneration and destruction of a joint is known as a neuropathic joint, neurogenic arthropathy, or a Charcot joint. The causative disease may be, for example, tabes dorsalis, diabetes mellitus, syringomyelia, congenital analgesia or a SCI. Regarding the mechanism, the 'trauma theory', which postulates that a joint is progressively destroyed as it is repeatedly subjected to a traumatic load in a painless, and therefore defenceless, state is advocated by many.

There are few reports on the development of a Charcot joint in the lower limbs due to spinal cord injury, but we have encountered such a case. The patient had sustained a fracture-dislocation of the 12th thoracic vertebra and a spinal cord injury in a cave-in accident in a coal mine 40 years previously and has since been assisted in walking by the aid of a short leg brace and a cane. Recently he developed a Charcot type joint in the right knee and right ankle, and the right ankle joint became infected with a refractory open wound, which necessitated a below-knee amputation.

\section{Case report}

A 65 year old male sustained a fracture-dislocation of the 12th thoracic vertebra and spinal cord injury in a cave-in accident while working in a coal mine in 1951. After about a year of hospitalisation, he was able to walk with the aid of crutches, and thereafter without braces. In 1985 , ulceration occurred at the medial malleolus of the right ankle joint. The ulcer was refractory and by August 1991 the ankle joint was involved, leading to pyogenic arthritis. Despite various therapies for the septic arthritis the wound did not heal and in January 1993 the patient was admitted to the department of orthopaedics at a university hospital where he was diagnosed as having a Charcot joint resulting from a spinal cord injury. He was referred to our department with the recommendation for an amputation in March 1993.

\section{Findings on initial examination}

The patient was alert, with no neurological abnormality of the upper limbs or trunk. Muscle testing revealed a slight laterality in favour of the left with the knee extensor/flexor showing grade 4 and the dorsiflexor/plantor flexor grade 0 . But even with this high level of injury, lumbar segment functions remained normal. Sensory functions had decreased from the first lumbar cord level distally, with analgesia from right lumbar dematomes distally. The right knee was swollen and had abnormal mobility and a sound friction was heard when the knee was moved passively. X-rays of the knee showed severe destruction and deformation.

The right ankle joint had an open wound and the inner surface of the capsule was villous due to the proliferation of synovia. X-rays revealed 

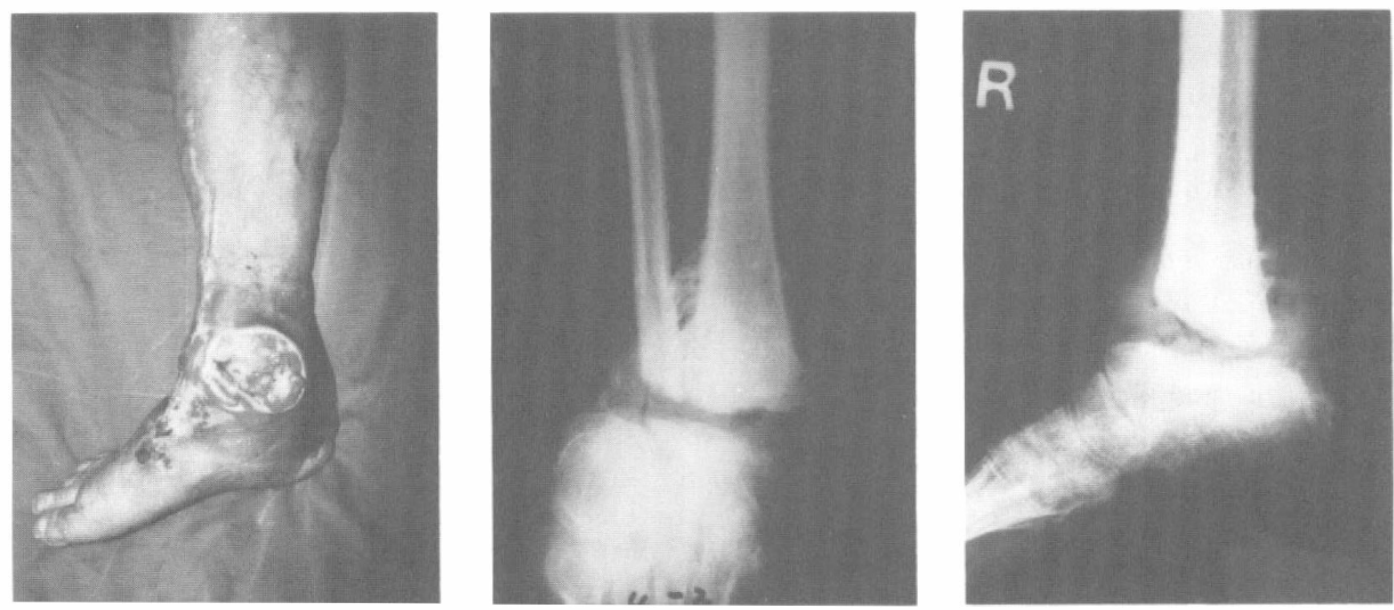

Figure 1 Right ankle joint.

a more severe degree of destruction compared to that seen in the knee joint (Fig 1).

The spine was deformed at the site of the fracture-dislocation but an MRI did not show evidence of syringomyelia (Fig 2). Our findings ruled out the possibility of other causes for the Charcot joints, such as tabes dorsalis and diabetes mellitus, and therefore a diagnosis of Charcot joint associated with spinal cord injury was made.

\section{Treatment}

Apart from amputation, arthrodesis was also considered in the treatment, but it was decided that a below-knee amputation and KondylenBettung-Munster (KBM) prosthesis were worth attempting. The factors taken into consideration were, among other things, (1) abnormal mobility of the knee joint, (2) decreased sensitivity in the weight-bearing region, such as the patellar ligament, and (3) delay in wound healing and ease of wounding.
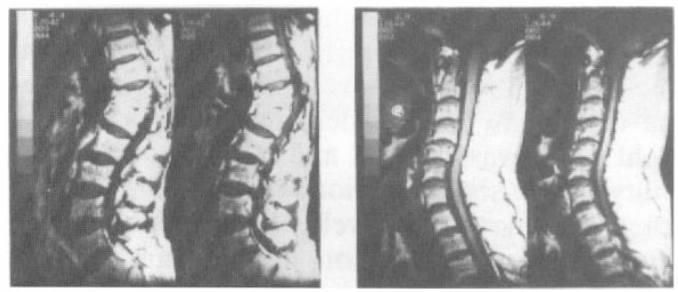

Figure 2 MRI revealed no signs of syringomyelia.
Pathological examination of the Charcot joint region showed a mixed destruction and proliferation, ie villous proliferation of the synovium and scattering of small bone fragments and cartilage fragments in the synovium. The articular cartilage showed degeneration, necrosis, and the proliferation changes of chondrocytes, all of which were, however, nonspecific.

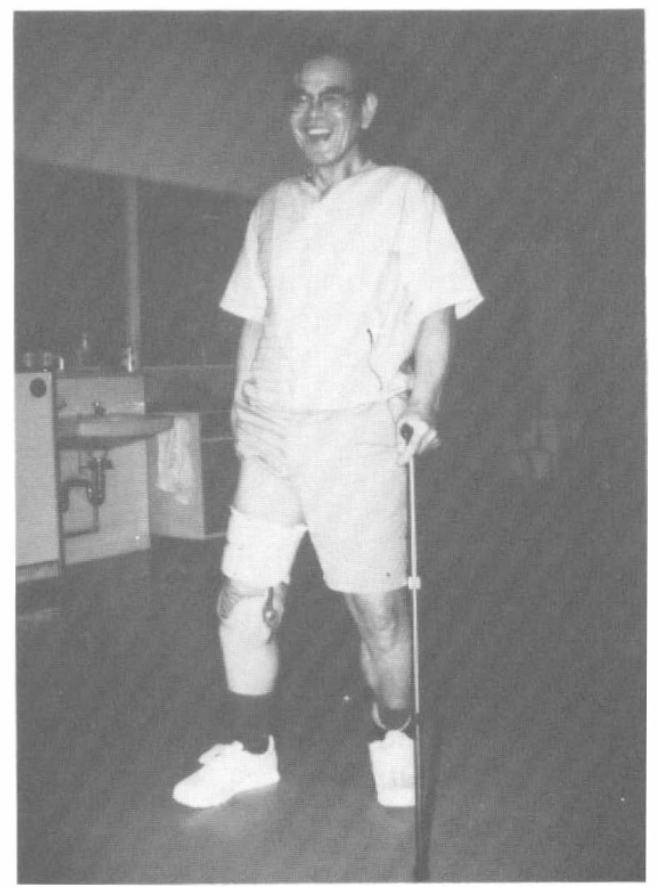

Figure 3 Right leg, conventional below-knee prosthesis. Left leg, ankle strap. 
Furthermore, wound healing was satisfactory. With maturation of the stump, a pylon KBM prosthesis was prepared and applied, and gait training was effected. However, the flail knee joint caused rotation of the stump in the socket, resulting in an abraded wound of the stump. Ultimately, the patient was able to walk with a conventional right leg prosthesis, a left ankle strap brace, and crutches. The patient said that he found it easier to walk now compared with the situation before the amputation. This was possible because the flail right knee joint and the dropfoot could now be controlled and the patient could also walk stably with a T-cane. (Fig 3).

\section{Conclusions}

The reported causes of Charcot joint are tabes dorsalis, diabetes mellitus, and syringomyelia, in order of decreasing frequency, but recent years have seen an increase in the frequency of Charcot joint associated with diabetes mellitus-especially of the foot and ankle joint.

Reports on Charcot joint due to spinal cord injury are rare and conservative therapies mainly depend on braces, and invasive therapies for immobilisation, which are not easy to perform. In order that immobilisation may be successful the following are essential: (1) selection of a proper immobilising position, (2) a sturdy internal fixation, (3) concomitant use of external fixation, and (4) positive protection against weightbearing until the completion of immobilisation. Finally, amputation may be necessary in refractory or infected cases. There is sensory impairment and the stump tends to become abraded with the wearing of a prosthesis.

\section{References}

1 Ijichi M (1982) Charcot joint. History, signs and symptoms, diagnosis and treatment. J UOEH 4: $185-198$.

2 Eichenholz SN (1966) Charcot Joints. C C Thomas, Springfield.

3 Samilson RL, Sankaran B (1959) Orthopedic management of neuropathic joints. Arch Surg 78: 115-121. 